\title{
Dharma-Samuccaya (éd. Lin), Chapitre VII (Kämajugupsā-Varga), 95-188: des éléments supplémentaires aux Appendices de M. de Jong *
}

\section{Yutaka Ojihara et Hideaki Nakatani}

95a. na tṛptir asti vișayair // (Leçon Lin).

- 95b. (Leçon dJ, définitive : api, anormalement au début, cf. Speijer, Skt. Syntax, $\S 423,2^{\mathrm{e}}$ alinéa.)

- 96d. (vinipātinah, Nom. pl. "ruineux, destructeurs".)

97c. nacet (SU, DS) teșām parityāgah, : “à moins qu'il n’y ait abandon total de ceux-là (=désirs),".

- 97d. kuryād dhy antaka utsavam (SU *suro 'tyanta-kausīdah*, DS *antaka utsāda*; Tib *bhūry andhā iva pātinah*?): "(en prévoyant de nouveaux butins,) la Mort fêterait son propre bonheur !" 98a. asya $\mathrm{d}^{\circ}$.

- 98c. tathânana-rasajña-sya (SU : Tib *tathā raso manojñaś ca*) : “de même, pour le connaisseur en saveurs (sis) dans la bouche (à savoir la langue)" // (Leçon $\mathrm{dJ}$ ).

98d. rasais tṛptir / (Leçon Lin).

99c. sa sukha ${ }^{\circ}$.

= 99d. gātrâmío (MS “śo tra sa', B "śro tra se'), “membre du corps".

$=100 \mathrm{~b}$. śrotram na syād vitṛptivat (ainsi Tib, sauf *asya* pour na syãt).

=100c. mano 'pi tṛptam vispașțair (B 'vi spāṃ șța') : cd. "quant à l'esprit, pour rassasié qu'il soit, il ne se rassasie point (dans la mesure où il s'agit d'acquérir) des Lois manifestes." (?)

-101a. șạ̣ indriyâśvāś capalā (aśva-, DS), "six chevaux-organes".

-101b. (Leçon $\mathrm{dJ}$, définitive: "(chevaux) dégagés de la bride qu'est la satisfaction".)

-102b. (Leçon Lin, définitive.)

-102c. analāyāḥ (SU d. *an-ālaya-ḥ*, Tỉ *analānām* : analā- comme surnom de trṣnā-, cf. ci-dessous 119d.): cd. "Voilà la nature propre de la Soif-feu, 
Dharma-Samuccaya (éd. Lin), Chapitre VII (Y. Ojihara - H. Nakatani)

(anala- 'feu' signifiant littéralement) 'à quoi fait défaut la satisfaction'." - A ce dernier propos, cf. M. Thieme, Kleine Schriften, p. 55sq., 709sq. : anala-, 'insatiable'.

-105c. sukhânusārako martyo (ainsi Tib: SU *sukhe 'nusārī kāmasya*?).

106b. satkriyāo (MS 'sa kri yā').

-106c. (Leçon dJ) / vicakșuṣko ? (MS “o kṣu sthä’).

106d. kāmavaśānugạ̣ (SU) / (Leçon Lin : Tib).

-107a. (Leçon dJ: SU, cf. Tib *asakṛ̂d vā citta-m*.)

-108c. (hitvā, "sans se soucier de...".)

-108d. kāmânusāriṇaḥ (anusārin-, Tib).

=111a. vanitā-bhoga- ${ }^{\circ}$, "fait de jouir de bien-aimées".

-111b. sukhaprāyeșu : abc. “qui ne s'attache pas aux désirs, qui, étant agréables de par le commerce sexuel, consistent largement en bonheur".

=113ab. ghana-cchāyāni rūpāṇi karma-pakvāṇi yāni... (Tỉ *karma(ṇo vi)pāka-*; SU *karmanā kāya-*?): "des signes (de bon augure) d'une coloration (aussi) dense (que celle du mont Ghana) et qu'ont parfaits les (bons) actes (de vie antérieure)". (?)

$=114 \mathrm{~b}$. na syād eva viyogitā. -114c. tathä ca (Tib).

-114d. vicāriṇaḥ (Tib) / (Leçon Lin: SU et DS).

=115a. (Leçon Lin, définitive: "(désirs) qui, étant inconstants, mènent avant tout au malheur". - Corriger SU「空」en「苦」.)

=115b. śūnyāś cânātmakā ya-m-eva : "et qui sont en soi vides, sans essence". (?)

117c. vivaśo / vaśago(Tib) nindāṃ(? MS 'ni tyaṃ') / vyathāṃ(ainsi SU: Tib *vitatha-*). -118c. (Leçon $\mathrm{dJ}$ ) // (Leçon Lin).

119b. saritas. $=119 \mathrm{~d}$. (Leçon $\mathrm{dJ}$, définitive : cf. ci-dessus 102c.)

-120a. jalasaṃbhava-mīnānāṃ (MS “o mī ne nah’).

120b. sacet tṛṣnā samā sadā (Tib *tṛṣnâsamā* : MS 'tṛ ṣñā su rah (?)') : ab. "si, (même) pour les poissons vivant dans l'eau, la soif demeure toujours la même (que pour n'importe quel être vivant)".

-122a. tṛptatâpi syāt (Tỉ *tṛptatā na syāt* : MS 'a tṛ pta syā pi’).

124b. cyavamānā yad anekaśah ( yad, Tib (c.), correspondant à c. tasmät : MS 
(3) Dharma-Samuccaya (éd. Lin), Chapitre VII (Y. Ojihara - H. Nakatani).

‘o mā ne șvu ne $\mathrm{ka}^{\circ}$ (?)’).

-124c. parais (Tib) / param (MS 'pa ra mam’) tapyanti viṣayais ?

125d. surāḥ // narāh. $\quad=126 \mathrm{~b}$ (Leçon dJ, définitive.)

-126d. tān budhaḥ parivarjayet (MS 'bu dhā' ; pari-, Tib).

127a. ${ }^{\circ}$ mūlam yatah s s : "Du fait que la tranquillité est (considérée - sc. b. mata- - comme étant) la racine du bonheur...".

127c. sadâsevyã ( $\bar{a}-\sqrt{ }$ sev-) / (Leçon Lin). 128a. ${ }^{\circ}$ rogãdi (b. bhayam).

-130ab. anekasukhasamsāre bhayam yad vidyate bhave (Tib *anekasukhe sam. prāpte bhayam yad bhavati bhayam*; MS “ ${ }^{\circ}$ sam srā vi yam yạ̣ hi vi dya te bha vet'): “Qu'il y ait danger dans l'existence qui passe par maints plaisirs, tout cela ...cd..."

=131ab. kașțair yair eva viṣayaih kṣapyante (SU: Tib *kșamante*) mūḍhacetasaḥ // tyaktuṃ ya eva kāmā na śakyante mūọhacetasām (lectio inferior, MS montrant un curieux mélange des deux leçons).

-132ab. ("Les hommes évitent toujours leur ennemi en craignant d'avoir à converser (avec lui)"?)

=133a. nirdāhakā ye viṣayā (SU : Tib *odāhah sadā vi*) // (Leçon dJ).

133b. bāliśānāṃ bhavanti, tān // (Leçon Lin).

134ab. ("Comme, dans la crainte du feu, d'aucuns honorent (=dẻifient ?) le feu luimême, de même ...cd...")

135cd. ("de même, voici un filet des plus terribles, (quoique) ravissant par les objets des sens (dont il est fait)": ayam, sc. saukhyam, V. 172 c. - stance qui précède dans le Sütra primordial.)

-136b. madhu- rāśi// rāga -mahodayah (SU *madhurâdi ${ }^{\circ}$ ), "grandement heureux de par le tas//charme de suc".

138a. ajñāno hi (a-jñāna-, W).

139a. mṛa-tṛṣā-m (Leçon dJ, qu'il faut maintenir sans la rayer).

139d. tathêdam (=137 d.).

141a. pratyakṣa-saukhyā viṣayā // pratyutpanna-sukhāḥ kāmā : ab. "Les objets des sens//Les désirs, où des plaisirs sont bel et bien visibles// présents , ne font à terme point de bien."

=141cd. rañjitā ... devā vikṣipta-manas-ạ̣ ... 
143b. abhyeyan nâvabudhyate (abhi-a- - $\mathbf{i}-$-, part. prés., Tib) // (Leçon Lin, lectio inferior, vidyate - DS *vijyate* — dans la valeur littéralement passive étant en somme insolite.)

143c. viṣaya-vyāhatair (Tib) / (Leçon Lin).

144a. jānīyād yady ayạ̣ devo (SU: MS 'jā nī ya rda dya me de vā (?)'; Tỉb *yatra yo*, pour yady ayam).

-144d. na kuryān (MS 'kū ryā.') ...(Leçon dJ) ...

145c. tathâpi mohito (Tib) / (Leçon Lin).

146b. devā vimohitāḥ (Tib) / (Leçon Lin). 146c. ...tān (sc. kāmān, cf. 145). -147a. ye caîtāṃs (?) tān praśamsanti (Tib: ye ca tãn $p^{\circ}$ ) / ye caîteșu (?) prasajyante (SU: $y e$ kāmeșu $\left.p^{\circ}\right)$ // ye viṣayeșu rajyante (ye, DS; MS 'vi șa ye șu tra ra kṣa nte').

=147b. teșāṃ duhkhhạ sadā sthitam (Tib) / teșām ādīnavo dhruvam (SU *te sadā devana-ruta-*?)? // (Leçon dJ).

148-149 (Stances yugma.)

-148a. vimohitāh prītyā ("troublés par une sensation d'amour") // vimohitâvidyā (Leçon Lin, lectio inferior en tant que les kāma n'y sont pas spécifiquement en jeu: de ce fait même, mieux vaudra y voir, et retenir tel quel, un comp. karmadh. quoique fort maladroit).

148c. vidyud-ulmuka-c ${ }^{\circ}$ (Tib *ulūkhala*, *()yantra-pātra*?) ?

149b. (vi-pra-lambhin- : MS “o lā bhi nah’, SU *olābhin-*; Tib *olopin-*, W; DS *lobhin-*.)

149c. (Référence tacite : anitya-, $148 \mathrm{~cd}$; dukha-, $148 \mathrm{ab}$; śúnya-, 149ab.)

\section{0-151 (Stances yugma.)}

-150c. tān yathāvad viditvā yo (B 'a ntya thā va dvi di kā yā'; yathãvat, Tỉb '( )ananyathātvāt', W).

-150d. na kāmeșu prasajyate (B 'n kā me șu pra sa hya te', MS 'o sa kṣa te').

152a. bhava-toyeșu (SU: DS ${ }^{* \circ}$ traya*) / (Leçon Lin: lectio inferior).

153c. vibhrașțo (toutes les versions?) / (Leçon Lin: MS).

153d. upapatsyate (Tib) / (Leçon Lin).

-154b. (vidyutā-, cf. BHSD, s. v. : thématisation à la moyen-indienne.)

156c. varjayanty aśivān. 
(5) Dharma-Samuccaya (éd. Lin), Chapitre VII (Y. Ojihara $\cdot$ H. Nakatani)

=157b. gāọhīkṛtyaîva (MS 'gā ḍhī kṛ tya va').

158b. rāga-tṛ̣̣nayā (Tib, $W$ : lectio superior) / (Leçon Lin).

-159c. abhavāya (DS *vi-bhāva*?) na vartante (SU: Tib *oāya pravart ${ }^{* *}$ ) // gacchanti.

160c. kāmān na seveta (MS 'kā mā nu se va cca').

161d. sampravartate ("se mettre en place").

-162a. tasmād yaḥ sabhayād vignaḥ (MS “o yaḥ su bha yā dve ti’; yah̆, que confirme DS; vigna-, $W:$ Tib) : ab. "celui qui s'alarme de ce monde tournant, dangereux océan de douleur".

-162c. 'viṣam ayam (ayam, correspondant à a. yah, malgré *viṣama(ka)* de toutes les versions) : cd. "voici qu'il abandonne totalement le mauvais poison qu'est la soif des désirs."

163b. ramyāṇi.

163d. (udaya-vyaya-, "revenus et dépenses, gain et perte")

-164c. śîlaśraddhạ (comp. dvand., type samāhāra). 164d. atyanta-śiva ${ }^{\circ}$.

165a. kāmajam dhanam apy, etad ( $W:$ Tib) : ab. "Un bénéfice né des désirs, s'il y en a, cela (n')est guide (que) pour la jungle d'existence."

165b. bhavâțavi-nidarśakam // saṃsārâțavi-deśakam.

-166a. yan na trāsayate 'pāyād (MS 'rbhrā śa ya te yo pā': SU et Tỉb *(saṃ) trāyate*): "en tant que ne pas faisant craindre sa diminution".

-166b. ("en tant que ne pas (spécialement) passant pour procurer le bien-être (matériel)".)

-166cd. mahaj jñānam ... nir-dhanam dhanam: "le grand savoir, et rien d'autre, s'appelle 'la richesse sans richesse'."

167d. 'gny-upasevakāh (SU: Tib *pra-sev**): "(mais) non ceux qui recourent au feu des désirs."

-168c. tṛ̣ṇā-homôpasamyuktā (MS 'tṛ ṣnā ma ho o': homa-, SU '(=)indhana') : "munis de l'oblation que constitue la soif".

170d. mṛtyur evâvaśișyate ("il ne reste (plus) que la mort") : seule leçon compatible, nous semble-t-il, avec le Gén. en série qui précède (Tib *mrtyurājâvas(ișy)ati*; de même, SU ?).

171d. kāma-sajjitaiḥ (Tib et DS *o saṃjñita-*): "(objets des sens) prêts (à en appeler) aux désirs". 
Dharma-Samuccaya (éd. Lin), Chapitre VII (Y. Ojihara - H. Nakatani)

-172c. buddha-gunâyuktā : a -yukta-, m.c. pour yukta-; double entente: "attachés (=dévoués) aux vertus du Buddha", en même temps que "munis. de la corde (=bride) du $B^{\circ "}$. =174a. (Leçon dJ, définitive.)

175c. pra-bhāngura - vi-śila- (SU *vi-sīma(n)* ?) : "très fraudeurs et sans moralité”. (?)

176a. ati-śīghreșu (Tib).

-178a. vidyullatâlātacakra-: ab. "comparables à l'éclair (passant) en zigzag, au tison (brandi) en cercle ainsi qu'au mirage".

=179a. (Leçon Lin, préférable, vu Mvy. 2826 qui fait partie d'une liste 'māyâdi-'.)

179b. gajakarṇy-upa ${ }^{\circ}$ : gajakarñi-, nom de plante (bulbeuse, partant sans noyau solide ?). (?)

-179c. nartakī-ranga-veșeșu (MS 'na dī ta ${ }^{\circ}$ : SU *na-rta-ka - rāga*) : "qui ressemblent à la mise en scène par les actrices" (cf. nața-rañga-, Mvy. 2837, au nombre également des mãya).

180c. māyopameșv anityeșu (MS ' 0 pa ma ni bhe șve șu (?)').

181a. dhāryante $(W)$ // vāryante. $\quad=182 \mathrm{a}$. viṣayair apakṛșyante.

-183c. (Leçon Lin, bien préférable.)

183d. (Leçon Lin) / narake pañca hetavaḥ (MS? - cf. a. vișayāh, de 5 sortes conformément aux pañcêndriya-?) ?

-185c. moham câśu pari ${ }^{\circ}$ (äśu, SU et Tib: DS *mohān doṣa*).

-186c. nirviṇno viṣayair dhīro $(W)$ // ${ }^{\circ}$ aih poṣo.

187b. 'doṣo hy avatīrṇa-tṛṣnah (MS 'ha ta vā rṇa').

=187c. sa dhīra-sattvo ... hatâgāh ( $W$ : dhĩra-, SU et Tib: MS 'vya ra'; āgas-, Tib: SU *āśá-*).

188a. ("qui, après avoir été sauvé de la boue des désirs, en est venu à vomir tous éléments vicieux (avalés et accumulés dan son corps)".)

188d. prasahya, "fortement" ou "instantanément".

* Suite de notre article dernièrement paru dans ce Journal même: Vol. XXX, No. 2 (March 1982), p. (1)-(6).

(Y. Ojihara, Professor, Kyoto University)

(H. Nakatani, Lecturer, Kobe-Gakuin University) 